\title{
Glutamine supplementation suppresses herpes simplex virus reactivation
}

\author{
Kening Wang, ${ }^{1}$ Yo Hoshino, ${ }^{1}$ Kennichi Dowdell, ${ }^{1}$ Marta Bosch-Marce, ${ }^{2}$ Timothy G. Myers, ${ }^{3}$ Mayra Sarmiento, ${ }^{1}$ Lesley Pesnicak, \\ Philip R. Krause, ${ }^{2}$ and Jeffrey I. Cohen ${ }^{1}$ \\ 'Laboratory of Infectious Diseases, National Institute of Allergy and Infectious Diseases, NIH, Bethesda, Maryland, USA. ${ }^{2}$ Center for Biologics Evaluation and Research, Food and Drug Administration, \\ Silver Spring, Maryland, USA. ${ }^{3}$ Research Technologies Branch, National Institute of Allergy and Infectious Diseases, NIH, Bethesda, Maryland, USA.
}

\begin{abstract}
Chronic viral infections are difficult to treat, and new approaches are needed, particularly those aimed at reducing reactivation by enhancing immune responses. Herpes simplex virus (HSV) establishes latency and reactivates frequently, and breakthrough reactivation can occur despite suppressive antiviral therapy. Virus-specific T cells are important to control HSV, and proliferation of activated T cells requires increased metabolism of glutamine. Here, we found that supplementation with oral glutamine reduced virus reactivation in latently HSV-1-infected mice and HSV-2-infected guinea pigs. Transcriptome analysis of trigeminal ganglia from latently HSV-1-infected, glutamine-treated WT mice showed upregulation of several IFN- $\gamma$-inducible genes. In contrast to WT mice, supplemental glutamine was ineffective in reducing the rate of HSV-1 reactivation in latently HSV-1-infected IFN- $\gamma$-KO mice. Mice treated with glutamine also had higher numbers of HSV-specific IFN- $\gamma$-producing CD8 T cells in latently infected ganglia. Thus, glutamine may enhance the IFN- $\gamma$-associated immune response and reduce the rate of reactivation of latent virus infection.
\end{abstract}

\section{Introduction}

Approximately $60 \%$ of people in the United States are infected with herpes simplex virus type 1 (HSV-1) and $20 \%$ with HSV-2. Prophylactic therapy with acyclovir or valacyclovir reduces the rate of cold sore (herpes labialis) recurrences by $40 \%-60 \%$ and the rate of genital herpes reactivation by $70 \%-80 \%$. Thus, other approaches to reduce reactivation of HSV are needed. Control of reactivation of HSV correlates with virus-specific T cells (1). Increased T cell function might reduce virus reactivation. Nutrients, including glucose and certain amino acids, are critical for T cell activation (2). Activated $\mathrm{T}$ cells require increased metabolism of glucose and glutamine for proliferation, and deprivation or inhibition of synthesis of these molecules reduces $\mathrm{T}$ cell proliferation $(3,4)$.

Glutamine serves as a nitrogen source for rapidly dividing cells including lymphocytes, in which it is important for energy production and for nucleotide synthesis. Mitogen-stimulated proliferation of peripheral blood mononuclear cells and secretion of IL-2 and IFN- $\gamma$ are dose-dependent on the level of glutamine (5). Glutamine is important for activation-induced proliferation of $\mathrm{T}$ cells $(3,6)$. Glutamine transporters are increased during $\mathrm{T}$ cell activation, and reduction of these transporters impairs $\mathrm{T}$ cell effector function $(6,7)$. Activation of naive $\mathrm{T}$ cells is associated with rapid uptake of glutamine, which requires the ASCT2 amino acid transporter (8). Activated human T cells require glutamine for produc-

Authorship note: $\mathrm{K}$. Wang and Y. Hoshino contributed equally to this work. Conflict of interest: The authors have declared that no conflict of interest exists. Submitted: June 10, 2016; Accepted: April 20, 2017.

Reference information: J Clin Invest. 2017;127(7):2626-2630.

https://doi.org/10.1172/JCl88990. tion of IFN- $\gamma$; depletion of glutamine inhibits $\mathrm{T}$ cell proliferation and reduces production of IFN- $\gamma$ and IL-2 $(6,9)$. Reduced availability of extracellular glutamine favors a Treg phenotype over a Th1 phenotype (10). Mice that receive glutamine have lower levels of HSV- 1 in vaginal fluid, higher titers of IFN- $\gamma$ in vaginal fluid, and increased numbers of activated CD8 cells in the spleen after HSV-1 infection (11). Glutamine deprivation and cellular stress have previously been shown to enhance replication of an HSV-1 mutant with deletion of virus infected cell polypeptide 0 (ICPO) (12). HSV-1 ICPO is critical for virus reactivation. These observations suggest that low glutamine levels might be associated with increased virus reactivation, or conversely that high levels might reduce reactivation. On the basis of these findings, we postulated that glutamine supplementation might increase $\mathrm{T}$ cell function and improve control of a chronic virus infection.

\section{Results and Discussion}

UV irradiation of the eyes of latently infected mice induces reactivation of HSV-1 from mouse trigeminal ganglia in vivo (13). Therefore, we infected mice with HSV-1 by corneal scarification, and 2 weeks later we supplemented drinking water with glutamine, glycine, or no supplement. After 2 weeks of supplement (4 weeks after infection), the latently infected animals were anesthetized, the eyes were irradiated with UV light, and 2 days later the animals were euthanized and their trigeminal ganglia were homogenized and assayed for infectious virus. This assay tests for virus already reactivated from the ganglia in vivo, since the tissue is homogenized immediately after dissection. The percentage of UV-induced HSV-1 reactivation in trigeminal ganglia from mice treated with glutamine was about half that of mice 
A
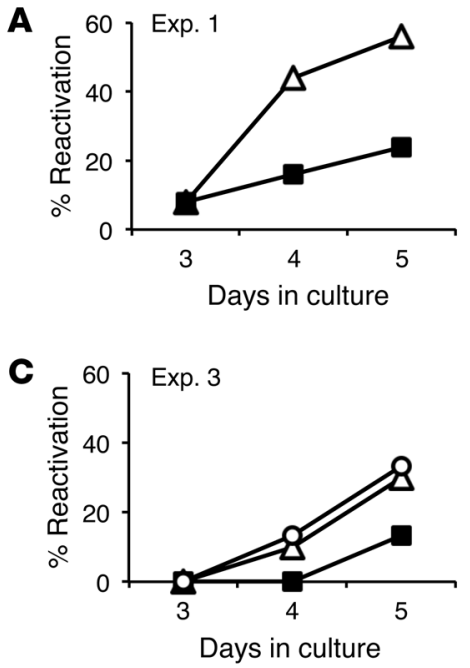
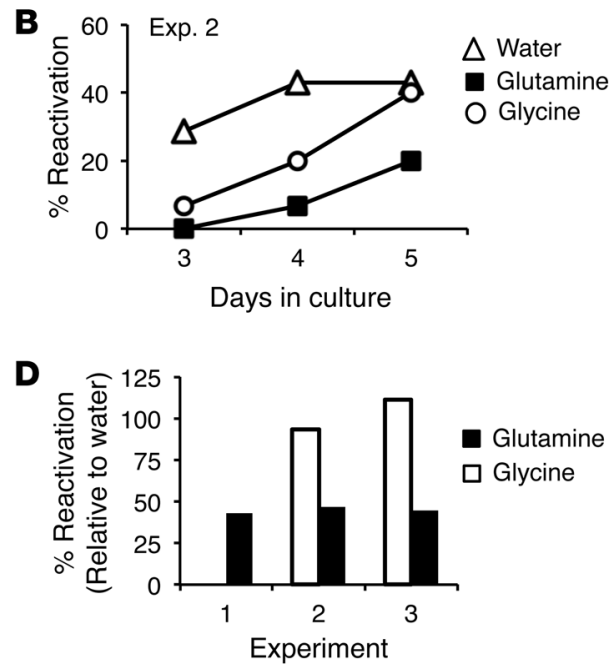

Figure 1. Reactivation of HSV-1 in trigeminal ganglia of mice receiving glutamine, glycine, or no supplement in their drinking water. Animals were inoculated with HSV-1 by corneal scarification, received supplemental amino acid 2 weeks later, and then, after an additional 2 weeks, were anesthetized and their eyes exposed to UV irradiation. Two days later, animals were euthanized, trigeminal ganglia were removed and homogenized, homogenates were plated onto Vero cell monolayers, and the number of ganglia with reactivated virus was determined. Three separate experiments were performed (A-C), and results at day 5 in culture for all 3 experiments are shown (D). Experiment 1 had 25 mice in each group, and experiments 2 and 3 had 15 mice each in the glutamine and glycine groups and 7 and 10 mice in the no supplement group, respectively. Fisher's exact test was used for statistics. treated with water or glycine in 3 independent experiments (Figure 1). The difference between glutamine and water was statistically significant in the first experiment $(P=0.042$, Fisher's exact test), but not in the second and third experiments, which had fewer animals; the difference was significant when the 3 experiments were pooled ( $P=0.0047$, Fisher's exact test). In contrast, the difference between glycine and water was not significant in any of the individual experiments or in the pooled experiment ( $P=0.47$, Fisher's exact test).

The standard animal model to assess spontaneous reactivation of HSV-2 is the guinea pig model. We infected guinea pigs intravaginally with $2 \times 10^{5} \mathrm{PFU}$ of HSV-2 (strain MS), monitored the animals for 2 weeks, and randomized the animals (based on similar acute disease scores) to receive either glutamine $(3 \mathrm{~g} / \mathrm{dl}$ in drinking water) or no supplement. Animals that received glutamine had fewer recurrences in 2 separate experiments during the 63-day treatment period (15-77 days after infection; Figure 2, $A$ and $B)$. In the first experiment, with 9 animals in each group, the difference was not significant $(P=0.093, t$ test; Figure $2 \mathrm{~A})$ for the area under the curve; however, in the second experiment, with 15 animals in each group, the difference was significant $(P=0.0322$, $t$ test; Figure 2B). When we pooled the data for these 2 treatment experiments, the difference was significant $(P=0.007)$. At the end of the treatment periods, all animals were taken off therapy (washout period), and while there was still a slight reduction in recurrences in animals that had received glutamine, the difference was diminished and not significant $(P=0.886, t$ test, Figure $2 \mathrm{C} ; P=0.645, t$ test, Figure 2D), and the differences were not significant when these data were pooled. Guinea pig vaginal swabs were obtained from 30 animals receiving supplemental glutamine or control, and HSV-2 DNA was determined by quantitative PCR. Only 1 of 60 samples from the glutamine-treated group was positive; in contrast, 2 of 60 samples from the glycine-treated group were positive, and 3 others were positive but below the standard curve (Supplemental Figure 1; supplemental material available online with this article; https://doi.org/10.1172/JCI88990DS1).

The HSV-1 latent viral load in mouse trigeminal ganglia (14) and the number of infiltrating CD8 T cells in ganglia both correlate with the likelihood of ex vivo reactivation $(14,15)$. Higher viral loads and lower numbers of CD8 T cells are associated with increased rates of reactivation. Therefore, we tested whether glutamine treatment of mice reduces the latent viral load in trigeminal ganglia or the number of infiltrating CD8 T cells. The latent HSV-1 DNA level in trigeminal ganglia of mice treated with glutamine was not significantly different from that of animals treated with glycine or water alone (Supplemental Figure 2A; $P \geq 0.14$ by Kruskal-Wallis test). The number of CD8 T cells infiltrating the ganglia in animals treated with glutamine was not significantly different from that in animals treated with glycine or water alone (Supplemental Figure 2B; $P=0.58$, Kruskal-Wallis test). Thus, the lower rates of reactivation observed in mice treated with glutamine were not due to lower latent HSV-1 load or an increase in the total number of CD8 $\mathrm{T}$ cells in the trigeminal ganglia.

To further analyze the mechanism whereby glutamine treatment reduces reactivation of $\mathrm{HSV}$ in vivo, we performed RNA microarray experiments comparing gene expression of latently HSV-1-infected mouse trigeminal ganglia for animals treated with supplemental glutamine and those treated with glycine. While diet-associated differences in gene expression were noted in 3 independent experiments (Supplemental Table 1; deposited in NCBI Gene Expression Omnibus, GSE95716), 5 genes were consistently upregulated in all 3 experiments (Table 1 and Supplemental Figure 3A) and no genes were consistently downregulated (Supplemental Figure 3B) in animals treated with glutamine versus glycine. Four of these five genes - IFN- $\gamma$-inducible protein 47 (Ifi47), chemokine ligand 9 ( $\mathrm{Cxcl9}$, also known as monokine induced by IFN- $\gamma[\mathrm{Mig}])$, IFN- $\gamma$-induced GTPase (Igtp), and $C d 274$ (also known as Pdl1) - are known to be induced by IFN- $\gamma$ and were expressed at higher levels in animals treated with glutamine and confirmed by quantitative reverse transcriptase PCR (Table 1). The fifth gene, protein disulfide isomerase associated 4 (Pdia4), has not been reported to have been tested for responsiveness to IFN- $\gamma$. Analysis of gene sets showed that sets of genes important for cellular responses to IFN and responses to microorganisms were consistently upregulated in latently HSV-1-infected ganglia of mice treated with supplemental glutamine versus glycine (Supplemental Figure 3C, Supplemental Figures 4-11, and Supplemental Table 2). 
A

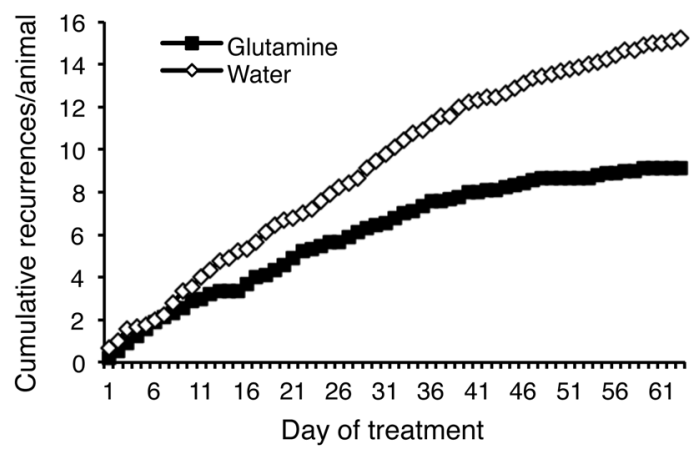

C

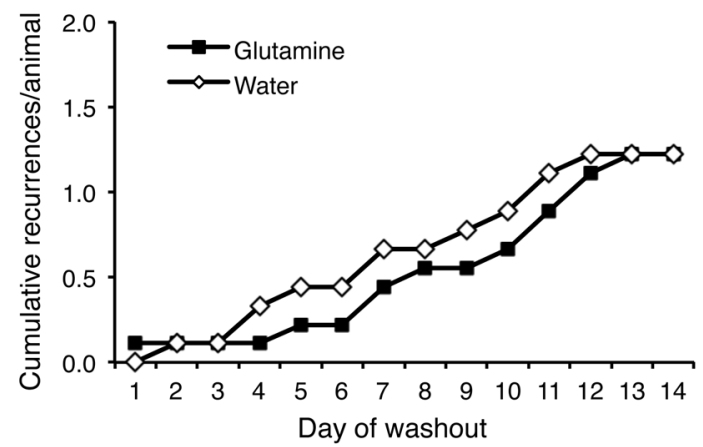

B

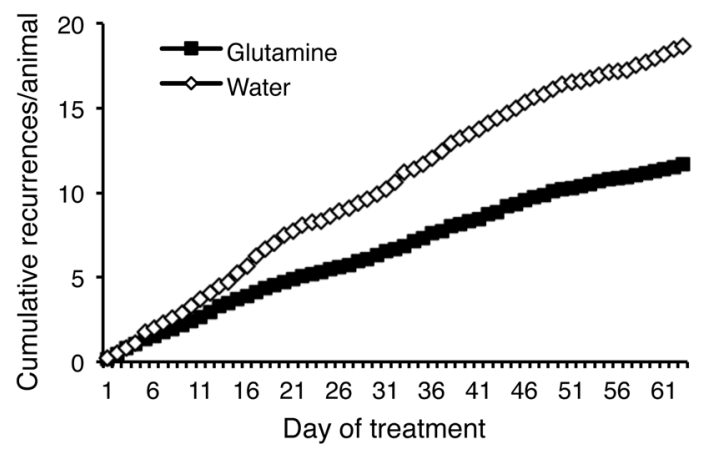

D

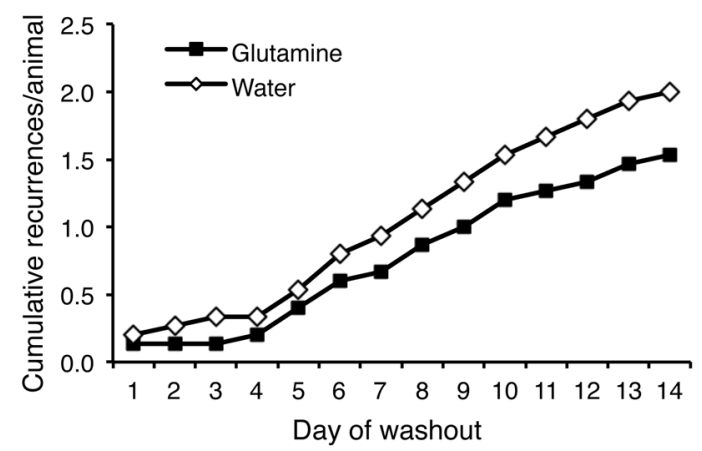

Figure 2. Rate of recurrences of genital HSV-2 in guinea pigs receiving glutamine or no supplement in their drinking water. Animals were inoculated intravaginally with HSV-2, and after recovery (2 weeks later on day 15) they were divided into 2 groups (based on lesion scores after acute infection) and glutamine was added to the drinking water of 1 of the 2 groups. Animals were monitored for recurrences, and disease scores were obtained each day; the mean number of cumulative recurrences per guinea pig in each group was plotted in each of 2 separate experiments (A and B). At the end of the treatment periods, all animals were taken off therapy (washout period) and monitored for recurrences in each of the 2 separate experiments (C and $\mathbf{D})$. Experiment 1 (A and $\mathbf{C}$ ) had 9 animals per group; experiment 2 (B and $\mathbf{D}$ ) had 15 per group. Two-tailed $t$ test was used for statistics.

Since glutamine increased the expression of IFN- $\gamma$-induced genes of latently infected mice, we tested whether mice unable to express IFN- $\gamma$ would fail to show a decrease in reactivation of HSV-1 after glutamine treatment. Ifng ${ }^{-/-}$mice (IFN- $\gamma$ knockout mice) were infected with HSV-1 and treated with glutamine or no supplement as described above. The frequency of reactivation was higher in IFN- $\gamma$ knockout mice compared with WT mice in the absence of supplemental glutamine, presumably because of the role of IFN- $\gamma$ in controlling the viral load and reactivation. Treatment of IFN- $\gamma$ knockout mice with glutamine had little effect on reducing the rate of reactivation compared with that in animals receiving no supplemental glutamine in 2 inde- pendent experiments (Figure 3, A and B), consistent with the observation that glutamine upregulates IFN- $\gamma$-inducible genes.

To further study the role of glutamine and IFN- $\gamma$ in preventing reactivation, we looked at HSV-specific IFN- $\gamma$-producing CD8 $\mathrm{T}$ cells in the ganglia, since these cells are thought to be important for preventing reactivation in the ganglia (16). Latently infected mice treated with glutamine had significantly higher numbers of HSV-specific IFN- $\gamma$-producing CD8 T cells in trigeminal ganglia than untreated animals $(P=0.007$, Mann-Whitney test) (Figure 3C), while the total number of CD8 T cells in treated and untreated groups was not significantly different $(P=0.375)$ (Figure 3D). These results, along with the observa-

\section{Table 1. Cellular genes upregulated in all 3 microarray assays in trigeminal ganglia of mice treated with glutamine}

Gene Description

Ifi47

[xcl9

Pdia4

Igtp

Cd274
IFN- $\gamma$-inducible protein 47

Chemokine (CXC) ligand 9 (monokine induced by IFN- $\gamma$ [Mig])

Protein disulfide isomerase associated 4

IFN- $\gamma$-induced GTPase

CD274 antigen (programmed death-ligand 1 [Pdl1])
mRNA expression ratio (Gln/Gly)

Microarray assay

$\begin{array}{cccc}\text { Exp. } 1 & \text { Exp. } 2 & \text { Exp. } 3 & \text { Exp. } 3 \\ 1.84 & 1.96 & 1.42 & 1.2 \\ 2.11 & 2.01 & 1.45 & 1.5 \\ 1.43 & 2.13 & 1.36 & \text { ND } \\ 1.88 & 2.22 & 1.46 & 1.7 \\ 1.97 & 2.10 & 1.36 & 1.4\end{array}$

ND, not done; qRT-PCR, quantitative reverse transcriptase PCR. 
A
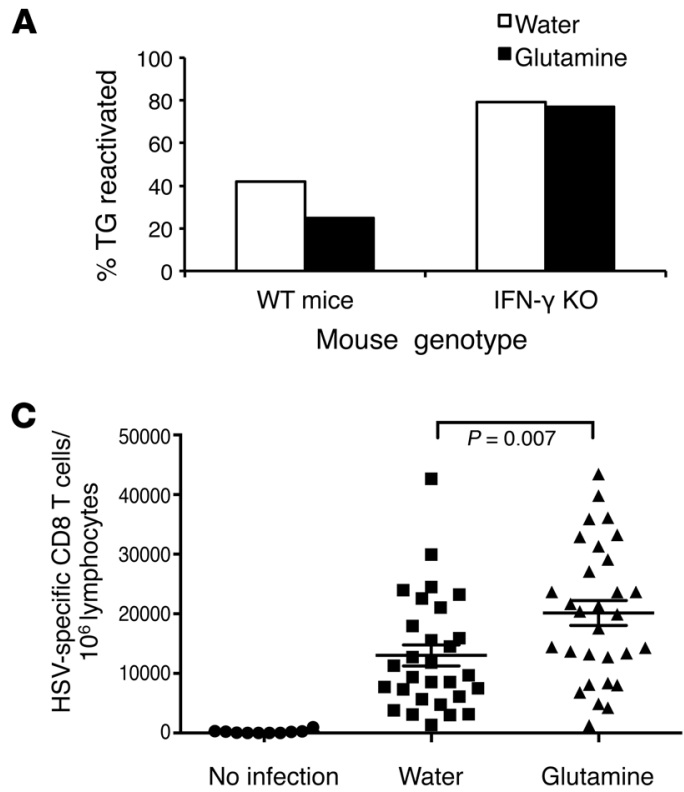

B

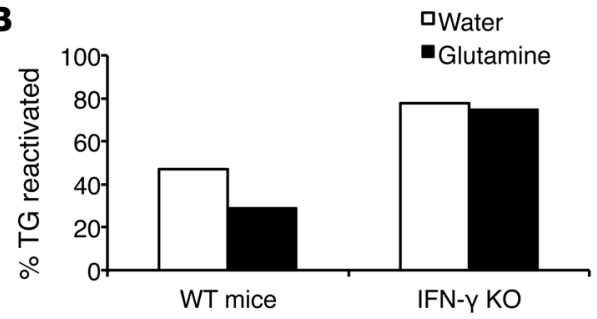

Mouse genotype

D

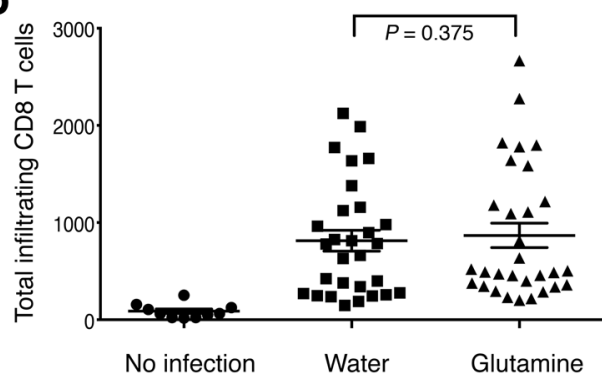

Figure 3. Reactivation of HSV-1 from trigeminal ganglia of WT and IFN- $\gamma$ knockout mice, and HSV-1-specific IFN- $\gamma$-producing CD8 T cells in WT mice receiving glutamine or no supplement. (A and B) Reactivation of HSV-1 from trigeminal ganglia (TC) of WT and IFN- $\gamma$ knockout mice receiving glutamine or no supplement in their drinking water. The first experiment had 10 WT mice and 11 IFN- $\gamma$ knockout mice in the glutamine group and 13 WT mice and 12 IFN- $\gamma$ knockout mice in the water group (A); the second experiment had 17 WT mice and 16 IFN- $\gamma$ knockout mice in the glutamine group and 15 WT mice and 16 IFN- $\gamma$ knockout mice in the water group (B). See legend to Figure 1 for experimental details. (C and D) Number of HSV-1-specific IFN- $\gamma$-producing CD8 T cells per $10^{6}$ lymphocytes (C) and total CD8 T cells per ganglion (D) of mice receiving glutamine or no supplement in their drinking water. The results of 4 independent experiments were pooled. Ten mice were used in the no infection group, and 30 each in the no treatment and glutamine groups. Mann-Whitney test was used for statistics; mean \pm SEM is shown.

tions in the IFN- $\gamma$ knockout mice, and the RNA microarray findings, support the hypothesis that glutamine acts through the IFN- $\gamma$ pathway to reduce HSV-1 reactivation. While glutamine could also affect virus replication, and therefore reactivation, we think this is less likely since prior studies show no effect of glutamine on replication of WT HSV in vitro (12).

IFN- $\gamma$ is important in controlling HSV reactivation. T cells in latently HSV-1-infected ganglia of humans and mice express IFN- $\gamma(17)$. IFN- $\gamma$ reduces reactivation from latency in several animal models. IFN- $\gamma$ is produced by CD8 T cells in cell suspensions from latently HSV-1-infected mouse trigeminal ganglia (18). Addition of IFN- $\gamma$ to latently infected trigeminal cell cultures reduces HSV-1 reactivation (18). IFN- $\gamma$ knockout mice have higher rates of HSV reactivation (19). We found that glutamine increased the level of several IFN- $\gamma$-inducible genes, including Cxcl9, which is critical for controlling genital HSV-2 by mobilizing virus-specific cytotoxic $\mathrm{T}$ cells to the nervous system and vagina in mice (20). Some studies question the importance of $\mathrm{T}$ cells for controlling reactivation since only a small portion of latently infected human neurons are surrounded by T cells; however, the number of activated T cells adjacent to HSV-infected neurons is thought to increase when low-level virus reactivation occurs in these neurons $(21,22)$.

Stress can result in glutamine depletion and impaired lymphocyte function. Stress also increases the rate of HSV reactivation (23). Stress can reduce the number of CD8 T cells in latently infected ganglia and the ability of HSV-1-specific CD8 T cells in ganglia to produce IFN- $\gamma$ in response to virus reactivation (23).
Therefore, glutamine may reverse the effects of stress on CD8 T cell responses and reduce reactivation.

While glutamine reduced the rate of in vivo HSV-1 reactivation in mouse trigeminal ganglia by only $50 \%$, this is the same level of effect that antiviral suppressive therapy has in reducing the rate of symptomatic recurrences of HSV-1 herpes labialis (24). In addition, while acyclovir partially reduces HSV-2 genital recurrences, it does not reduce the 2- to 3-fold increased risk of HIV acquisition associated with HSV-2 (25). Thus, there is a clear need for other therapies to suppress oral and genital HSV recurrences. The ability of glutamine to reduce HSV reactivation in 2 different animal models suggests a new approach to reduce reactivation of the virus in humans.

\section{Methods}

Statistics. All statistics except microarray were done in JMP 7.0.2 (SAS Institute); $P$ less than 0.05 was considered significant. Microarray data normalization and differential expression were computed using SAS and JMP/Genomics 4.0 (SAS Institute).

Study approval. All animal experiments were performed under protocols approved by the Animal Care and Use Committees of the National Institute of Allergy and Infectious Diseases and the Food and Drug Administration. A complete description of methods is provided in Supplemental Methods.

\section{Author contributions}

YH, KW, KD, MS, LP, MBM, and PRK performed the animal studies. KW and YH did real-time PCR. KD quantified virus-specific 
CD8 cells in ganglia. TGM performed the microarray experiments and analyses. JIC, YH, and KW designed the study. JIC, YH, KW, TGM, and PRK wrote the paper.

\section{Acknowledgments}

This research was supported by the intramural research programs of the National Institute of Allergy and Infectious Diseases and the
Center for Biologics Evaluation and Research. This paper is dedicated to the memory of Priscilla Schaffer, who inspired this work.

Address correspondence to: Jeffrey I. Cohen, Building 50, Room 6134, 50 South Drive, National Institutes of Health, Bethesda, Maryland 20892-8007, USA. Phone: 301.496.5265; Email: jcohen@niaid.nih.gov.
1. Zhu J, et al. Immune surveillance by $\mathrm{CD} 8 \alpha \alpha^{+}$ skin-resident $\mathrm{T}$ cells in human herpes virus infection. Nature. 2013;497(7450):494-497.

2. Buck MD, O'Sullivan D, Pearce EL. T cell metabolism drives immunity. JExp Med. 2015;212(9):1345-1360.

3. Wang R, et al. The transcription factor Myc controls metabolic reprogramming upon $\mathrm{T}$ lymphocyte activation. Immunity. 2011;35(6):871-882.

4. Macintyre AN, et al. The glucose transporter Glut1 is selectively essential for CD4 T cell activation and effector function. Cell Metab. 2014;20(1):61-72.

5. Chang WK, Yang KD, Shaio MF. Effect of glutamine on Th1 and Th2 cytokine responses of human peripheral blood mononuclear cells. Clin Immunol. 1999;93(3):294-301.

6. Carr EL, et al. Glutamine uptake and metabolism are coordinately regulated by ERK/MAPK during $\mathrm{T}$ lymphocyte activation. J Immunol. 2010;185(2):1037-1044.

7. Sinclair LV, Rolf J, Emslie E, Shi YB, Taylor PM, Cantrell DA. Control of amino-acid transport by antigen receptors coordinates the metabolic reprogramming essential for $\mathrm{T}$ cell differentiation. Nat Immunol. 2013;14(5):500-508.

8. Nakaya M, et al. Inflammatory $\mathrm{T}$ cell responses rely on amino acid transporter ASCT2 facilitation of glutamine uptake and mTORC1 kinase activation. Immunity. 2014;40(5):692-705.

9. Hörig H, et al. Exogenous glutamine requirement is confined to late events of $\mathrm{T}$ cell activation. JCell Biochem. 1993;53(4):343-351.

10. Klysz D, et al. Glutamine-dependent $\alpha$-ketogluta- rate production regulates the balance between $\mathrm{T}$ helper 1 cell and regulatory $\mathrm{T}$ cell generation. Sci Signal. 2015;8(396):ra97.

11. Uyangaa E, Lee HK, Eo SK. Glutamine and leucine provide enhanced protective immunity against mucosal infection with herpes simplex virus type 1. Immune Netw. 2012;12(5):196-206.

12. Bringhurst RM, Dominguez AA, Schaffer PA. Glutamine deprivation causes enhanced plating efficiency of a herpes simplex virus type 1 ICPOnull mutant. J Virol. 2008;82(22):11472-11475.

13. LeBlanc RA, Pesnicak L, Godleski M, Straus SE. The comparative effects of famciclovir and valacyclovir on herpes simplex virus type 1 infection, latency, and reactivation in mice. J Infect Dis. 1999;180(3):594-599.

14. Hoshino Y, Pesnicak L, Cohen JI, Straus SE. Rates of reactivation of latent herpes simplex virus from mouse trigeminal ganglia ex vivo correlate directly with viral load and inversely with number of infiltrating CD8+ T cells. J Virol. 2007;81(15):8157-8164.

15. Liu T, Khanna KM, Chen X, Fink DJ, Hendricks RL. CD8(+) T cells can block herpes simplex virus type 1 (HSV-1) reactivation from latency in sensory neurons. JExp Med. 2000;191(9):1459-1466.

16. St. Leger AJ, Hendricks RL. CD ${ }^{+}$T cells patrol HSV-1-infected trigeminal ganglia and prevent viral reactivation. JNeurovirol. 2011;17(6):528-534.

17. Khanna KM, Bonneau RH, Kinchington PR, Hendricks RL. Herpes simplex virus-specific memory $\mathrm{CD} 8^{+} \mathrm{T}$ cells are selectively activated and retained in latently infected sensory ganglia. Immunity. 2003;18(5):593-603.
18. Liu T, Khanna KM, Carriere BN, Hendricks RL. Gamma interferon can prevent herpes simplex virus type 1 reactivation from latency in sensory neurons. JVirol. 2001;75(22):11178-11184.

19. Minami M, et al. Role of IFN-gamma and tumor necrosis factor-alpha in herpes simplex virus type 1 infection. J Interferon Cytokine Res. 2002;22(6):671-676.

20. Shin H, Iwasaki A. A vaccine strategy that protects against genital herpes by establishing local memory T cells. Nature. 2012;491(7424):463-467.

21. Held K, et al. Expression of herpes simplex virus 1-encoded microRNAs in human trigeminal ganglia and their relation to local T-cell infiltrates. JVirol. 2011;85(19):9680-9685.

22. van Lint AL, Kleinert L, Clarke SR, Stock A, Heath WR, Carbone FR. Latent infection with herpes simplex virus is associated with ongoing CD8+ T-cell stimulation by parenchymal cells within sensory ganglia. JVirol. 2005;79(23):14843-14851.

23. Freeman ML, Sheridan BS, Bonneau RH, Hendricks RL. Psychological stress compromises $\mathrm{CD} 8^{+}$ T cell control of latent herpes simplex virus type 1 infections. JImmunol. 2007;179(1):322-328.

24. Gilbert SC. Suppressive therapy versus episodic therapy with oral valacyclovir for recurrent herpes labialis: efficacy and tolerability in an open-label, crossover study. J Drugs Dermatol. 2007;6(4):400-405.

25. Celum C, et al. Effect of aciclovir on HIV-1 acquisition in herpes simplex virus 2 seropositive women and men who have sex with men: a randomised, double-blind, placebo-controlled trial. Lancet. 2008;371(9630):2109-2119. 